\title{
Menopause and Gynecological Malignancy
}

\author{
Sabera Khatun, Jannatul Ferdous
}

\begin{abstract}
Menopause is a consequence of biological aging. Menopause does not cause cancer, but the risk of developing cancer increase as a woman ages. Treatment of cancer can also cause premature menopause. Among the gynecological cancer, cervical cancer tops the list of common cancer. Postmenopausal women are prone to have persistent human papillomavirus (HPV) infection. There is no role of primary prevention of cervical cancer by vaccination in postmenopausal women. Secondary prevention by Paps smear only may provide false negative result as the cervix is often flushed with the vagina. So, scraping by Ayres spatula and using endometrial brush is necessary in these women. Unfortunately, symptoms of cervical cancer in postmenopausal women are nonspecific and associated with comorbidities. So, any symptom of cervical cancer in postmenopausal women should be properly evaluated. Ovarian cancer is the number one cancer of death among all gynecologic malignancies. Majority of the ovarian cancer in postmenopausal women present with vague symptom and have the advance stage at prevention. Currently, no reliable screening test is available. Yearly routine pelvic examination, transvaginal ultrasonography and serum CA-125 can be performed in selective high-risk women only. Endometrial cancer is the most common gynecologic cancer in the USA. Most endometrial carcinomas are diagnosed at an early stage and have a good prognosis. Though vulvar and vaginal cancer are rare malignancies, yet these are prevalent in postmenopausal women. Any postmenopausal women with long-term intense itching, pervaginal discharge or any ulcer of vulva or vagina should be evaluated properly.
\end{abstract}

Keywords: Menopause, Malignancy, Cervix, Ovary, Endometrium.

How to cite this article: Khatun S, Ferdous J. Menopause and Gynecological Malignancy. J South Asian Feder Menopause Soc 2013;1(2):75-79.

\section{Source of support: Nil}

Conflict of interest: None declared

\section{INTRODUCTION}

\section{Menopause and Cervical Cancer}

Menopause means cessation of menstruation for at least 1 year with declining ovarian hormonal activity. According to one of the hospital statistics in Bangladesh the age range of menopause is 38 to 62 years, average 52 years. Menopause does not cause cancer, but the risk of developing cancer increases as a woman ages. Women who have gone through natural menopause are more likely to develop cancer because they are older. In addition, cancer treatment can cause menopause and menopausal symptoms.
Commonly, the incidence of female genital organ malignancy increases with age excepting ovarian and cervical cancer which has two peak age incidences. First peak age is 35 years and second peak incidence is at 55 years. Cancer of the cervix is very rare under the age of 25 years, mostly occurring in 55 to 60 years age group. The progression from high grade changes to cancer cervix takes between 1 and 30 years with an average of 10 years. ${ }^{1}$

In addition to general risk factors another risk factor for development of cervical cancer in postmenopausal women is that they are prone to have persistent human papillomavirus (HPV) infection. A study done at the College of Public Health, University of Lowa, USA. They examined 105 women aged 45 to 64 annually for 7 years to evaluate HPV in cervical cytological specimens. The cumulative prevalence of HPV was 34 and 24\% had HPV high-risk oncogenic types which are associated with genital cancers. The persistence rate of HPV infection was $16 \%$. They concluded that postmenopausal women are infected with persistent oncogenic HPV at a substantial rate, supporting the need for continued screening in postmenopausal women to detect preneoplastic genital lesions. $^{2}$

On the contrary, postmenopausal women who take hormones combining estrogen and progestin have cellular abnormalities detected in the cervix more frequently than nonusers of hormone therapy do, but the risk of precancerous lesions and cervical cancer does not differ between users and nonusers, according to a report based on 6 years of data from the Women's Health Initiative. ${ }^{3}$

Most studies on cervical cancer screening have focused on premenopausal women, with little information provided on the perimenopausal or postmenopausal (PMP) population. However, these women remain at increased risk for cervical cancer given that there is a secondary peak in prevalence of high-risk HPV subtypes in this older population, ${ }^{4}$ and that certain high-risk HPV subtypes persist to a greater extent in this population. ${ }^{5}$ Unfortunately, many of these older women have not had regular access to gynecologic care, or cervical cancer screening. Of menopausal women who were diagnosed with invasive cervical cancer, $50 \%$ of women were over age 65 who had not had a Pap smear in the past 3 years. ${ }^{6}$ Approximately, one quarter of elderly women have never been screened by Pap testing. ${ }^{7}$ 
Cervical cancer is a preventable disease. There is no role of primary prevention of cervical cancer by vaccination in postmenopausal women. Secondary prevention by Pap's smear creates some problem in postmenopausal women because of some factors:

a. Cervix is flashed with the vaginal vault and squamocolumnar junction is receded inside the endocervical canal. So, scraping by Ayre's spatula only provides false negative result. To avoid this fallacy Pap's smear in a menopausal lady should always be taken both by Ayre's spatula and endocervical brush. Because many cases of cervical cancer are diagnosed in women older than 50, Pap's smear needs to be continued even after menopause.

b. Colposcopic examination of cervix in a menopausal lady also poses some problem as most of the time it is difficult to expose the cervix and unsatisfactory colposcopic examination is the rule. To avoid these false negative results, endocervical curettage should always be associated with colposcopic examination. For the same reason, VIA as a method of screening for cervical cancer in menopausal women is not ideal.

Most common symptom of cervical cancer is abnormal and irregular pervaginal bleeding, often in the form of postcoital bleeding in a sexually active woman. In sexually inactive menopausal women, these symptoms are generally nonexistent. Therefore, proper screening is imperative. In premenopausal women, periods become irregular, sometimes heavier and sometimes lighter. Most women assume this abnormal or unusual bleeding is a normal part of menopause, which it can be but could also be a sign of cervical cancer. So, any symptom of cervical cancer should be checked. Unfortunately because symptoms of cervical cancer after menopause are nonspecific, we see a rise in number of women battling this disease. Other symptoms of cervical cancer after menopause could be pelvic pain, increased vaginal discharge and postcoital bleeding.

Pyometra as a complication of cervical cancer is common finding in menopausal women. Not only complication, pyometra may create difficulty in treatment also. Because before radical hysterectomy or radiotherapy, pyometra needs to be drained out, which is also very difficult as the cervical os is stenosed and tight in a menopausal woman.

Another special complication of invasive cervical cancer in menopausal woman is osteoporosis. A group of investigators in Japan measured bone mass density (BMD) in 50 patients with invasive cervical cancer without bone metastasis and compared with these in 50 control women. Their preliminary results suggest that patients with invasive cervical cancer have a lower BMD, resulting increased risk of osteoporosis.

Through squamous cell carcinoma is the most common histological type of cervical cancer, in a postmenopausal woman, nonsquamous rare histological types may occur. Adenoid cystic carcinoma usually occurs in postmenopausal black women of high parity. ${ }^{8,9}$ Clean cell adenocarcinoma occurs in two distinct age groups. Those younger than 24 years and those older than 45 years. ${ }^{10}$ Mean age at diagnosis of small-cell carcinoma are 49 years and 52 years. The second peak of papillary serous carcinomas of cervix is 65 years. Sarcomas are also common in menopausal age. The mean age of diagnosis of malignant mixed Mullerian tumor (MMMT) of cervix is 49.6 years. Average age of diagnosis of Verrucous carcinoma is 51 years. Malignant melanoma of cervix has in general been reported to occur in seventh and eighth decade of life. Management of cervical cancer of a postmenopausal woman needs some special considerations. These are:

a. Most of them are of advanced stage disease - so always needs examination under general anesthesia.

b. Most of them are diabetic, hypertensive, anemic with some cardiac and cerebrovascular problem. So, needs referral to these specific subspecialties.

c. Women over 70 years cannot tolerate the toxicities of radiotherapy and chemotherapy. Moreover, they cannot bear the burden of radical hysterectomy. So, they need special consideration for their management.

d. Advancing age is one of the important prognostic factors for 5 years survival of a patient of cervical cancer.

\section{Menopause and Ovarian Cancer}

Ovarian cancer is the 8 th most common cancer and the 7th most common cause of cancer death in women worldwide. ${ }^{11}$ A woman's risk at birth of having ovarian cancer some time in her lifetime is nearly $1.5 \%$, and the risk of dying from ovarian cancer is almost $1 \%$. Approximately, $30 \%$ of ovarian neoplasms in postmenopausal women are malignant, whereas only about $7 \%$ of ovarian epithelial tumors in premenopausal patients are frankly malignant. ${ }^{12}$

\section{Age Distribution of Ovarian Malignancy}

Approximately, $1.2 \%$ were diagnosed under age $20 ; 3.6 \%$ between 20 and $34 ; 7.4 \%$ between 35 and $44 ; 18.6 \%$ between 45 and $54 ; 23.4 \%$ between 55 and $64 ; 20.1 \%$ between 65 and $74 ; 17.6 \%$ between 75 and 84 ; and $8.1 \%$ $85+$ years of age (Graph 1).

From 2005 to 2009, the median age at diagnosis was 63. For the same period, the median age of death from ovarian cancer was $71 . .^{13}$ 


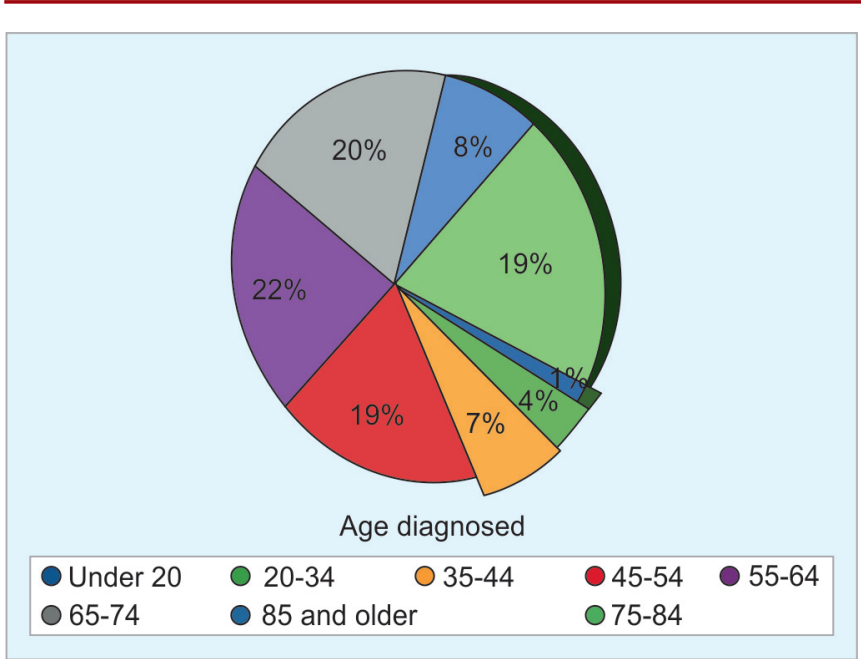

Graph 1: Approximate age at diagnosis from years 2002-2006

Approximately, $90 \%$ of ovarian cancers are derived from cells of the coelomic epithelium or modified mesothelium. Approximately 75 to $80 \%$ of epithelium cancers are of the serous histologic type. Less common types are mucinous $(10 \%)$, endometrioid $(10 \%)$, clear cell, Brenner and undifferentiated carcinoma, each of the latter three representing less than $1 \%$ of epithelial lesions. ${ }^{12}$

Once a woman enters menopause, there is an expectation that the ovaries become inactive. While they do have a lower level of activity, they are still capable of producing cysts. With the increased use of imaging and the recognition by primary care doctors that ovarian cancers present with subtle symptoms, more ovarian masses are being detected in postmenopausal women. Currently, no reliable screening test is available. Yearly, routine pelvic examination is the most convenient and easily applicable method. But, it should be done in combination with other tests like transvaginal ultrasonography, serum CA-125 and color flow Doppler imaging study. Screening for ovarian cancer should be selective for:

a. Peri- and postmenopausal women.

b. Age more than 50 years.

c. Nulliparous women with history of PCOS.

d. Women with family history of ovarian cancer.

e. Women with personal history of breast and colon cancer.

f. Women with BRCA I and BRCA II gene mutations.

In women with a BRCA I or BRCA II mutation, the risk of ovarian and breast cancer may be as high as 54 and $82 \%$ respectively. ${ }^{14}$ An estimated 1 in 600 US women carries the BRCA I or BRCA II gene mutation. ${ }^{15}$ Women who appear to be at high risk for ovarian cancer should be offered genetic testing for BRCA I and BRCA II and positive women who have completed their family should be recommended to undergo prophylactic bilateral salpingooophorectomy.
In screening studies, 5 to $20 \%$ of women over the age of 50 with no other symptoms will have an ovarian mass detected on ultrasound with simple ovarian cysts less than $5 \mathrm{~cm}$, the risk of an ovarian cancer is very small (0-1\%). In a large study conducted at the University of Kentucky, no women with simple ovarian cysts less than $10 \mathrm{~cm}$ in diameter developed ovarian cancer. However, 10 to $40 \%$ of complex cysts with solid areas and papillations will harbor a malignancy. Ovarian cysts are common in postmenopausal women. Simple cysts less than $5 \mathrm{~cm}$ in diameter without concerning features can safely be followed with repeated ultrasounds. Other ovarian masses should be referred to gynecologic oncologists for appropriate surgery, which may include laparoscopic removal of the ovaries with staging procedure if necessary. ${ }^{16}$

Postmenopausal women who use estrogen-only replacement therapy are at increased risk for ovarian cancer, especially after 10 or more years of use. The risk of malignancy index (RMI), first described by Jacobs in 1990, is one method of differentiating between benign and malignant masses. ${ }^{17}$ Utilization of this index may facilitate better triage of suspicious pelvic masses to gynecologic oncologists. The RMI incorporates the menopausal status, an ultrasonic score and the serum CA-125 level. The diagnosis of an ovarian cancer requires an exploratory laparotomy. The ovarian tumor should be removed intact, if possible, and a frozen histologic section obtained. If ovarian malignancy is present and tumor is apparently confined to the ovaries or the pelvis, then thorough surgical staging should be carried out. The surgical management of all patients with advanced-stage disease is approached in a similar manner, with modifications made for the overall status and general health of the patient as well as the extent of residual disease present. If the patient is medically stable then a primary optimum debulking or cytoreductive surgery which includes total omentectomy, bilateral pelvic lymphadenectomy, total abdominal hysterectomy is done. Sometimes hepatectomy, splenectomy, bowel resection is required. These operations pose great burden to menopausal women and require special attention.

Regarding adjuvant therapy, no adjuvant chemotherapy is recommended for low-risk early stage disease. But for high risk stage I epithelial ovarian cancer treatment with carboplatin and paclitaxel chemotherapy for three to six cycles is used in most patients, although single-agent carboplatin may be preferable for frailer menopausal women.

Neoadjuvant chemotherapy might be appropriate in selected menopausal patients who are at high risk for operative morbidity and mortality, e.g. those with significant cardiac disease or with large pleural effusion. But, primary 
cytoreductive surgery should be considered the standard of care for most patients.

Five-year survival rate of ovarian cancer in menopausal women is much lower $(15 \%)$ than the younger one $(40 \%){ }^{18}$

\section{Menopause and Endometrial Cancer}

Endometrial cancer is the most common gynecologic cancer in the United States, affecting 21 of every 100,000 women, according to the National Cancer Institute's (NCI) Surveillance, Epidemiology, and End Results (SEER) Program. ${ }^{19}$ Developing countries and Japan have incidence rates four to five times lower than Western industrialization nations, with the lowest rates being in India and South Asia. $^{20}$

Over the past two decades, the rate of endometrial cancer has decreased by more than $26 \%{ }^{19}$ This may be because many women are entering menopause who previously used combination oral contraceptives (OCs) containing progestin, which is associated with a reduced risk of endometrial hyperplasia and cancer. The increased use of progestin in hormone replacement therapy (HRT) regimens in recent years also likely plays a role.

Risk factors for endometrial cancer are conditions typically associated with chronic elevations of endogenous estrogen levels or increased estrogen action at the level of the endometrium. These include obesity, history of chronic anovulation, diabetes mellitus, estrogen-secreting tumors, exogenous estrogen unopposed by progesterone or progestin, tamoxifen use, and a family history of Lynch type II syndrome (hereditary nonpolyposis colorectal, ovarian or endometrial cancer). Since endometrial cancer may occur in the absence of risk factors, however, they should not be the sole means of identifying patients.

Two different clinicopathological subtypes of endometrial cancer are recognized: the estrogen-related (type I, endometrioid), and the nonestrogen-related (type II, nonendometrioid). Approximately, $80 \%$ of newly diagnosed endometrial carcinomas in the western world are endometrioid in type. ${ }^{21}$ The average age of patients with endometrioid cancer is approximately 63 years, and 70\% are confined to the corpus at the time of diagnosis. Their 5 -year survival is approximately $83 \% .^{22}$ By contrast, the average age of patients with nonendometrioid cancer is 67 years. And, at least half have already spread beyond the corpus at the time of diagnosis. Their 5-year survival is approximately $62 \%$ for clear cell carcinoma and 53\% for papillary serous cancers. $^{22}$

Most endometrial carcinomas are diagnosed at an early stage and have a good prognosis. The most common presenting symptom is postmenopausal bleeding. As there is no evidence to support routine screening for endometrial cancer, effort should be directed toward making women and their carers aware that postmenopausal bleeding, however slight, is abnormal and deserves prompt investigation. Because most patients with endometrial carcinomas present early, it is unlikely, based on current technology, that a population screening program would be of any value. ${ }^{23}$

Endometrial evaluation is called for when any menopausal woman not taking HRT develops uterine bleeding after more than 1 year of amenorrhea. It is also indicated in any postmenopausal woman on HRT for 6 months or more with persistent uterine bleeding, and any previously amenorrheic woman on HRT who begins bleeding without apparent cause.

The measurement of endometrial thickness by transvaginal sonography (TVS) is now almost standard in the evaluation of postmenopausal bleeding. However, screening asymptomatic women for endometrial cancer with TVS is not recommended. Although the test is very specific, it is not sensitive. Many women without endometrial cancer will have an endometrial thickness of $5 \mathrm{~mm}$ or more.

Endometrial biopsy is another option for patients with postmenopausal bleeding, though the American College of Obstetricians and Gynecologists (ACOG) does not recommend this method for screening asymptomatic menopausal women not on HRT. ${ }^{24}$

The primary treatment of endometrial cancer is surgery. The initial approach for all medically fit patients should be total abdominal hysterectomy and bilateral salpingooophorectomy. Removal of a vaginal cuff is not necessary. The adnexa should be removed because they may be the site of microscopic metastases. In addition, patients with endometrial carcinoma are at increased risk for ovarian cancer. Such tumors sometimes occur concurrently. Surgical staging including lymphadenectomy, should be performed in those patients listed below:

1. Patients with grade 3 lesions.

2. Patients with grade 2 tumors $>2 \mathrm{~cm}$ in diameter.

3. Patients with clear cell or papillary serous carcinomas.

4. Patients with greater than $50 \%$ of myometrial invasion.

5. Patients with cervical extension.

Special problem with these patients are obesity and cardiac disease. Cardiac problem demands inclusion of cardiac anesthesiologist during operation. Prolonged use of aspirin and heparin needs special consideration.

Regarding adjuvant therapy, though adenocarcinomas are less sensitive to radiotherapy, addition of radiotherapy as adjuvant increases the disease free interval, though it does not improve the 5 years survival rate.

\section{Menopause and Vulva/Vaginal Cancer}

These are rare malignancy of postmenopausal women. Chronic itching and discharge are the most common symptoms. So, any postmenopausal woman complaining 
of long-term itching and discharge per vagina should have a wedge or a Keyes biopsy specimen which usually can be taken in the office under local anesthesia.

Any ulcer, growth, induration, swelling or color change of vulva/vagina should have similar type of evaluation.

\section{CONCLUSION}

Menopause is an unavoidable change that every women will experience, assuming she reaches middle age and beyond. Menopause does not cause cancer, but the risk of developing cancer increases as a woman ages. Commonly, the incidence of female genital organ malignancy increase with the ages. Among the gynecological cancer, cervical cancer tops the list of common cancer followed by ovarian cancer, endometrial cancer and other rare vulvar or vaginal cancer. Most of these postmenopausal women are not aware of these malignancies and their symptoms. Unfortunately, symptoms are also nonspecific and most women assume these abnormalities as a normal part of menopause. So, we see a rise in number of women battling these diseases. So, increasing the awareness, continued screening and prompt evaluation and treatment is imperative in postmenopausal women.

\section{REFERENCES}

1. Managing menopause. Available from: www.managingmenopause.org.au/health-issues.

2. Smitha EM, Johnson SR, Ritchiec JM, Feddersen D, Wangd D, Turekd LP, Haugend TH. Persistent HPV infection in postmenopausal age women. Int J Gynaecol Obstet 2004; 87(2):131-137.

3. Yasmeen $\mathrm{S}$, et al. Incidence of cervical cytological abnormalities with aging in the women's health initiative. Obstet Gynecol 2006;108(2):410-419.

4. Castle PE, Jeronimo J, Schiffman M, et al. Age-related changes of the cervix influence human papillomavirus type distribution. Cancer Res 2006;66(2):1218-1224.

5. Castle PE, Schiffman M, Herrero R, et al. A prospective study of age trends in cervical human papillomavirus acquisition and persistence in Guanacaste, Costa Rica. J Infect Dis 2005; 191(11):1808-1816 view at Publisher, view at google scholar, view at PubMed, View at scopus.

6. Celentano DD, Klassen AC, Weisman CS, Rosenshein NB. Cervical cancer screening practices among older women: results from the Maryland cervical cancer case-control study. J Clin Epidemiol 1988;41(6):531-541 view at scopus.

7. Cornelison TL, Montz FJ, Bristow RE, Chou B, Bovicelli A, Zeger SL. Decreased incidence of cervical cancer in medicareeligible California women. Obstet Gynecol 2002;100(1): 79-86.

8. Hensley ML, Blessing JA, Mannel R. Fixed-dose rate gemcitabine plus docetaxel as first-line therapy for metastatic uterine leiomyosarcoma: a Gynecologic Oncology Group (GOG) phase II trial. Gynecol Oncol 2008;109:329-334.

9. Omura GA, Blessing JA, Major E, Silverberberg S. A randomized trial of adriamycin versus no adjuvant chemotherapy in stage I and II uterine sarcomas. J Clin Oncol 1985;9: $1240-1245$

10. Clement PB, Young RH. Mesenchymal and mixed epithelialmesenchymal tumors of the uterine corpus and cervix. In: Clement PB, Young RH, editors. Atlas of gynecologic surgical pathology. Philadelphia: WB Saunders; 2000. p. 177-210.

11. GLOBOCAN 2008.Available at: http://globocan.iarc.fr. Accessed July 2012.

12. Scully RE, Yong RH, Clement PB. Tumors of the ovary, maldeveloped gonads, fallopian tube and broad ligament. In: Atlas of tumor pathology. 3rd series, fascicle 23. Washington, DC: Armed Forces Institute of Pathology; 1998. p. 1-168.

13. Ovarian cancer statistics. Ovarian Cancer National Alliance. Available from: www.ovariancancer.org/about-ovarian-cancer/ statistics.

14. King MC, Marks JH, Mandell JB. New York Breast Cancer Study Group. Breast and ovarian cancer risks due to inherited mutations in BRCA 1 and BRCA 2. Science 2003;302:643-646.

15. Cancer gene mutation linked to earlier menopause. Reuters; 2013 January 30 .

16. Holtz D. Women's health source: ovarian cysts and masses in menopause. Available at: www.mainlinehealth.org/oth/ Page.asp?PageID=OTH004241.

17. Jacobs I, Oram D, Fairbanks J, Turner J, Frost C, Grudzinskas JG. A risk of malignancy index incorporating CA-125, ultrasound and menopausal status in the preoperative diagnosis of ovarian cancer. Brit J Obstet Gynecol 1990;97:922-929.

18. Neijt JP, ten Bokkel Huinink WW, van der Burg MEL, van Oosteron AT, Willeme PH, Heintz AP, et al. Randomized trial comparing two combination chemotherapy regimens (CHAP-5 versus $(\mathrm{CP}$ ) in advanced ovarian carcinoma: a randomized trial of the Netherlands joint study group for ovarian cancer. J Clin Oncol 1987;5:1157-1168.

19. National Cancer Institute. Endometrial cancer. Available at: http://www3.cancer.gov/admin/fmb/2001 sigitems/ endometrial.html. Accessed on: August 26, 2002.

20. Parazzini F, La Vecchia C, Bocciolone L, Franceschi S. The epidemiology of endometrial cancer. Gynecol Oncol 1991;41: 1-16.

21. Part J, Gallardo A, Cuatrecasas M, Catasus L. Endometrial carcinoma: pathology and genetics. Pathology 2007;39:1-7.

22. Creasman WT, Odicino F, Mausinneuve P, Quinn MA, Beller U, Benedet JL, et al. Carcinoma of the corpus uteri. FIGO Annual Report, Vol 26. Int J Gynaecol Obster 2006;95(suppl 1): S105S143.

23. Robertson G. Screening for endometrial cancer. Med J Aust 2003;178(12):657-659.

24. American College of Obstetricians and Gynecologists. Routine cancer screening. Committee Opinion \#247. Washington, DC: ACOG; Dec 2000.

\section{ABOUT THE AUTHORS}

\section{Sabera Khatun (Corresponding Author)}

Professor and Head, Department of Obstetrics and Gynecology, Division of Gyne-oncology, Bangabandhu Sheikh Mujib Medical University Dhaka, Bangladesh, e-mail: sabera120khatun12@yahoo.com

\section{Jannatul Ferdous}

Associate Professor, Department of Obstetrics and Gynecology Division of Gyne-oncology, Bangabandhu Sheikh Mujib Medical University, Dhaka, Bangladesh 